\title{
FP01
}

\section{Laboratory Measurements of Seismic Anisotropy in Synthetic Silica Cemented Sandstones with Controlled Fracture Geometry}

\author{
P. Tillotson* (National Oceanography Centre, Southampton), J. Sothcott \\ (National Oceanography Centre, Southampton), A.I. Best (National \\ Oceanography Centre, Southampton), M. Chapman (University of \\ Edinburgh) \& X.Y. Li (British Geological Survey)
}

\section{SUMMARY}

In this study we present ultrasonic laboratory measurements of P- \& S-wave velocity and attenuation on novel, synthetic, "fractured" (penny shaped voids), sandstone samples saturated with three different viscosity pore fluids. The synthetic sandstone was found to be a very realistic analogue for natural, silicacemented, porous sandstones, with the advantage of having a controlled fracture geometry. Comparison of the shear-wave splitting results to the fracture density of the rock (obtained from X-ray CT image analysis), confirms that shear-wave splitting is a robust measure of fracture density in high porosity, silicacemented, sandstones and is independent of the saturating fluid viscosity. 


\section{Introduction}

Extracting geological information from seismic data requires the use of equivalent-medium theories. These theories are used to estimate fracture and fluid properties within reservoir rocks. For this reason, validation of such theories is of utmost importance.

In this study we present laboratory measurements on a novel, synthetic, "fractured" (penny shaped voids), sandstone samples (Figure 1) saturated with three different viscosity pore fluids. The synthetic sandstone was found to be a very realistic analogue for natural, silica-cemented, porous sandstones, with the advantage of having a controlled fracture geometry. Comparison of the shear-wave splitting results to the fracture density of the rock (obtained from X-ray CT image analysis), confirms that shear-wave splitting is a robust measure of fracture density in high porosity, silica-cemented, sandstones and is independent of the saturating fluid viscosity.

\section{Laboratory results}

Synthetic sandstone samples were analysed using helium porosimetry for porosity and grain density, nitrogen permeametry for Klinkenberg corrected permeability, X-ray diffraction for mineral composition and SEM imaging for an estimate of grain size and sorting. Fractured cores were X-ray CT scanned to obtain fracture size, density and distribution. SEM imaging showed well sorted sand grains (mean diameter $120 \mu \mathrm{m}$ ) and a relatively even distribution of cement between the grains. XRD analysis showed that the composition of the whole rock was $>90 \%$ Silica $\left(\mathrm{SiO}_{2}\right)$. The porosity $(\approx$ $30 \%$ ), permeability $(\approx 30 \mathrm{mDarcy})$ and grain density $\left(2590 \mathrm{~kg} / \mathrm{m}^{3}\right)$ are comparable with natural high porosity sandstones. Image analysis of X-ray CT scan data was used to calculate the fracture density $(\varepsilon)$ of the fractured sample. The rock samples were measured air dry, saturated with de-aired deionized distilled water and saturated with glycerin.

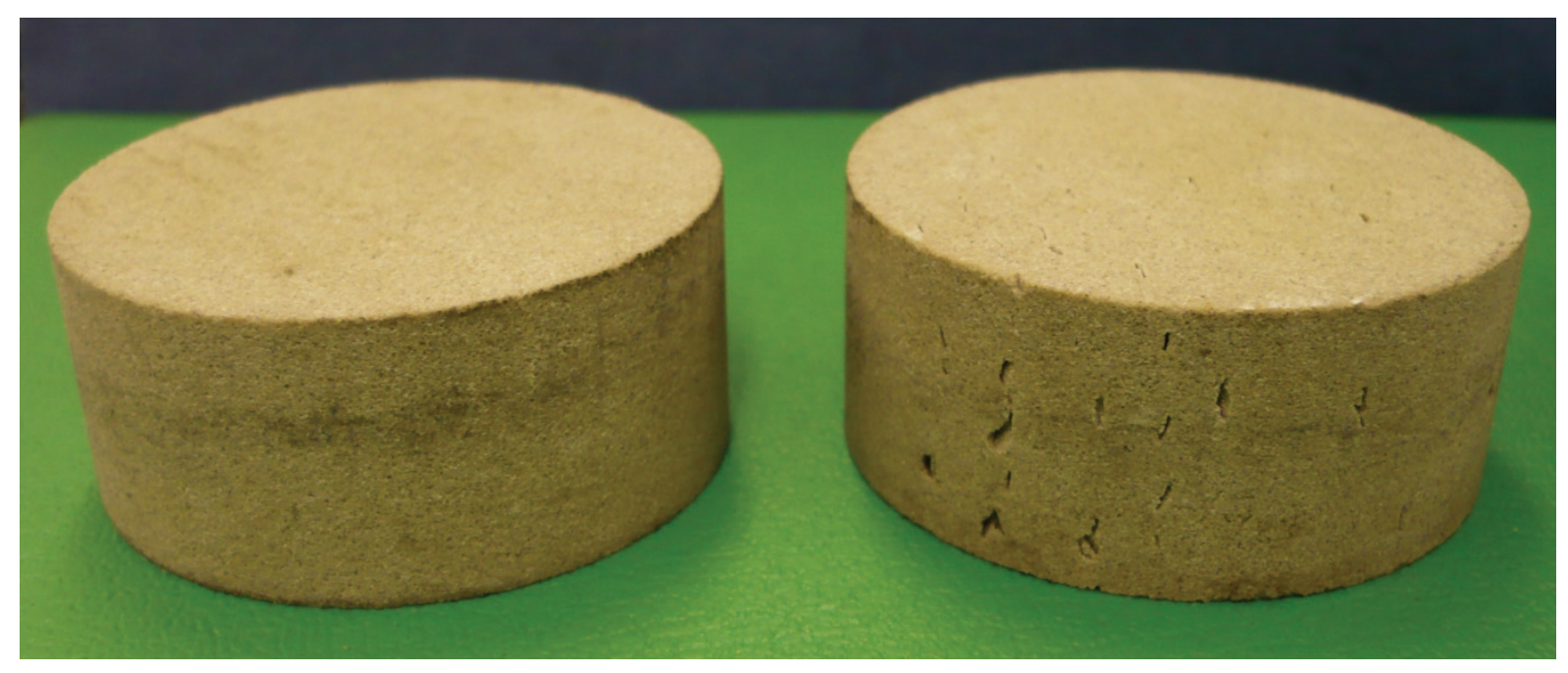

Figure 1: Blank synthetic silica-cemented sandstone sample (left) and corresponding synthetic sample with vertically aligned penny-shaped voids (right).

Initial results in Figure 2 using the ultrasonic pulse-echo system confirm that shear-wave splitting is a robust estimate of fracture density and agrees with theory that predicts that fracture density should be $100 \varepsilon$ and independent of saturating fluid viscosity (Hudson 1981). This is in agreement with previous studies carried out on less realistic synthetic rocks (Ass'ad et al. 1992, Rathore et al. 1995, Tillotson et al. 2010). 
a)

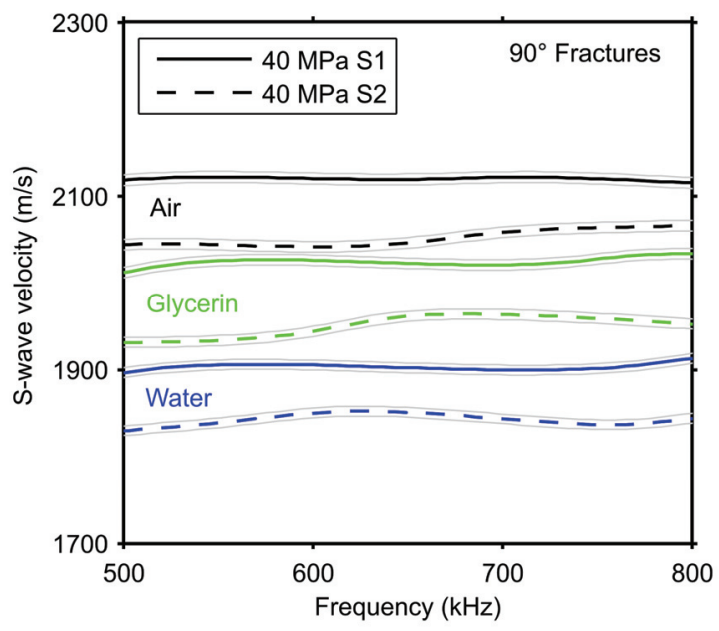

b)

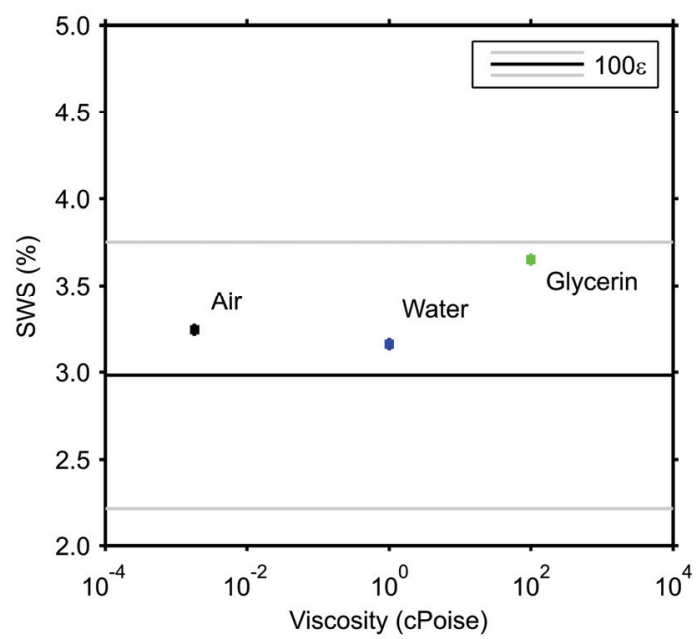

Figure 2: (a) Shear-wave velocities (S1 \& S2) versus measurement frequency at $40 \mathrm{MPa}$ effective pressure in the $90^{\circ}$ sample with penny-shaped voids (i.e., wave propagation at $90^{\circ}$ to fracture normal) when saturated with air, water and glycerin. (b) Shear-wave splitting versus fluid viscosity. The fracture density with error bars measured from X-ray CT image analysis is indicated (i.e., 100 times fracture density).

\section{Conclusions}

Laboratory validation of theoretical models of seismic wave propagation in fractured porous media requires synthetic rocks with controlled fracture properties; the fractures should closely resemble the idealised shapes and distributions used in various theoretical models. To date there have only been a handful of published experiments of this nature, but all suffer from results on somewhat unrealistic synthetic rocks. Experimental ultrasonic results on our newly developed and realistic synthetic silica cemented sandstones with meso-scale, penny-shaped voids further confirm that shear wave splitting is a good indicator of fracture density in porous rocks, regardless of pore fluid type.

\section{Acknowledgements}

The authors with to thank the UK's Natural Environmental Research Council and Sinopec for providing financial support for this work. This work forms part of the $\mathrm{PhD}$ studies of Philip Tillotson under a NERC-BGS PhD studentship.

\section{References}

Ass'ad, J. M., Tatham, R. H. and McDonald, J. A. [1992] A physical model study of microcrackinduced anisotropy. Geophysics, 57(12), 1562-1570.

Hudson, J. A. [1981] Wave speeds and attenuation of elastic waves in material containing cracks. Geophysical Journal of the Royal Astronomical Society, 64, 133-150.

Rathore, J. S., Fjaer, E., Holt, R. M. and Renlie, L. [1995] P- and S- wave anisotropy of a synthetic sandstone with controlled crack geometry. Geophysical Prospecting, 43, 711-728.

Tillotson, P., Chapman, M., Best, A., Sothcott, J., McCann, C., Shangxu, W. and Li, X. Y. [2010] Observations of fluid-dependent shear-wave splitting in synthetic porous rocks with aligned penny-shaped fractures. Geophysical Prospecting, doi: 10.1111/j.1365-2478.2010.00903.x. 